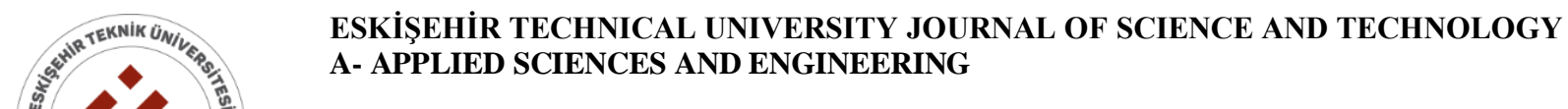

$2019,20(4)$, pp. $446-457$, DOI: 10.18038 /estubtda. 514207

\title{
MODIFICATION OF CLEMENTS' METHOD FOR ASSESSING THE CAPABILITY OF A NON-NORMAL PROCESS WITH AN APPLICATION
}

\author{
Burcu AYTAÇOĞLU ${ }^{1, *}$, Cemil Giray GENÇ² \\ ${ }^{1}$ Department of Statistics, Faculty of Science, Ege University, İzmir, Turkey \\ 2 Department of Statistics, Faculty of Science, Ege University, İzmir, Turkey
}

\begin{abstract}
In many industries process capability studies are conducted in order to determine the capability of the process to produce acceptable products and it is one of the important activities of statistical process control. In order to express the capability of a process, process capability indices are frequently used. However, they are usually computed under the assumption that the process data follow a normal distribution. Normality assumption may be violated and the use of these traditional capability indices may cause misleading interpretation about the capability of the process. One of the most widely discussed methods to handle non-normality is Clements' method which was proposed in 1989. Clements' method uses the Pearson family of curves for calculating capability indices for any shape of distribution. It requires the estimation of the mean, standard deviation, skewness and kurtosis and makes use of the classical estimators of skewness and kurtosis. In this study, we discussed the use of more robust estimators of skewness and kurtosis in the calculation of process capability index by Clements' method. For this purpose, capability indices with the use of these robust estimators are computed by simulation and the mean square errors of them are reported. The comparison is done through simulating Weibull and lognormal data with several different parameter values. Finally, a real life application to oil pump manufacturing in automotive industry is presented.
\end{abstract}

Keywords: Process Capability Index, Non-normality, Statistical Process Control, Skewed Population, Robust Estimator

\section{INTRODUCTION}

Process capability analysis is the activity of analyzing process variability relative to product specifications and it helps manufacturing in decreasing variability and improving quality. Process capability indices (PCIs) are frequently used to quantify the capability of the process in producing items within the specifications. The most widely used indices in industry; $\mathrm{C}_{\mathrm{p}}$ and $\mathrm{C}_{\mathrm{pk}}$ were defined as

$$
\begin{gathered}
\mathrm{C}_{\mathrm{p}}=\frac{\mathrm{USL}-\mathrm{LSL}}{6 \sigma} \\
\mathrm{C}_{\mathrm{pk}}=\min \left\{\mathrm{C}_{\mathrm{pu}}, \mathrm{C}_{\mathrm{pl}}\right\}=\min \left\{\frac{\mathrm{USL}-\mu}{3 \sigma}, \frac{\mu-\mathrm{LSL}}{3 \sigma}\right\}
\end{gathered}
$$

where LSL and USL denote the lower and upper specification limits, respectively. Since the process mean $\mu$ and variance $\sigma^{2}$ are unknown they are estimated from the sample.

The traditional PCIs are usually computed and interpreted under the assumption that the process data follow a normal distribution. However, normality assumption may be violated and the use of these indices may cause misleading interpretation about the capability of the process.

Several studies are available in literature that account for non-normality of the process data. There are usually two ways to handle a non-normal process data. The one is to transform non normal data to normal data and to use standard PCIs given in (1) and (2). Numerous normalizing transformations were proposed in the literature to use for this purpose. (see, for example, Box and Cox [1], Johnson and Kotz [2], Ryan [3], Ryan and Schwertman [4], Niaki and Abbasi [5]). The other is to use PCIs defined directly for nonnormal data. Clements [6] used the Pearson family of curves for calculating capability indices for any

*Corresponding Author: burcu.aytacoglu@ege.edu.tr

Received: 17.01.2019 Published: 30.12.2019 
shape of distribution. Liu and Chen [7] have modified Clements' method using the Burr XII distribution. Wu et al. [8] proposed weighted variance PCIs which are based on the semivariance approximation of Choobineh and Branting [9]. Chang et.al.[10] computed PCIs which are based on weighted standard deviation method. In addition, there are also several comparative studies for the proposed methods of PCI calculation.(see, for example, Tang and Than [11], Kovarik and Sarga [12], Sennaroglu and Senvar [13], Hosseinifard et al. [14]). Authors may also benefit from the review papers by Kotz and Johnson [15], Anis [16], Wu et al. [17].

In this study, we considered Clements' method [6] which is the most widely discussed methods to handle non-normality in capability index calculations. It requires the estimation of the mean, standard deviation, skewness and kurtosis and makes use of the classical measures of skewness and kurtosis. We modified the Clements' method by using the alternative measures of skewness and kurtosis some of which are more robust than the classical measures. These are Bowley coefficient of skewness $\left(s k_{2}\right)$, Groeneveld and Meeden coefficient of skewness $\left(s k_{3}\right)$, Pearson coefficient of skewness $\left(s k_{4}\right)$, Moors coefficient of kurtosis $\left(k r_{2}\right)$, the centered Hogg coefficient of kurtosis $\left(k r_{3}\right)$, and the centered coefficient of kurtosis suggested by Crow and Siddiqui [18] $\left(k r_{4}\right)$. They are briefly discussed in Section 3. The robustness of these measures was studied by Kim and White [19]. They investigated the robustness of the measures by adding a single outlier to the generated data and also by considering mixture distribution. We use the measures which are available in Kim and White [19] in the context of Clements' method. For comparative purposes, Weibull and lognormal data with several different parameter values were generated and mean square errors (MSEs) of the estimators of capability indices were simulated. These distributions were considered since they are frequently encountered in industrial processes when the process is not symmetric and they are the most discussed asymmetric distributions in process capability studies.

This article is organized as follows. In Section 2, the datails of the Clements' method is explained. In Section 3, the measures of skewness and kurtosis which are used in this study are discussed. In Section 4, the simulation procedure is explained and the MSEs are reported. In Section 5, a real life application to oil pump manufacturing in automotive industry is presented and some concluding remarks are given in Section 6.

\section{CLEMENTS' METHOD}

Clements [6] used the Pearson family of curves for calculating capability indices for any shape of distribution. In the Clements' method $6 \boldsymbol{\sigma}$ in (1) was replaced by $\mathbf{U}_{\mathbf{p}}-\mathbf{L}_{\mathbf{p}}$, giving

$$
\mathbf{C}_{\mathbf{p}}=\frac{\mathrm{USL}-\mathbf{L S L}}{\mathbf{U}_{\mathbf{p}}-\mathbf{L}_{\mathbf{p}}}
$$

where $\mathbf{U}_{\mathbf{p}}$ and $\mathbf{L}_{\mathbf{p}}$ denote 99.865 and 0.135 percentiles, respectively. The process mean $\mu$ in (2) was estimated by the median $\mathbf{M}$, and $3 \boldsymbol{\sigma}$ was replaced by $\mathbf{U}_{\mathbf{p}}-\mathbf{M}$ for finding $\mathbf{C}_{\mathbf{p u}}$ and by $\mathbf{M}-\mathbf{L}_{\mathbf{p}}$ for finding $\mathbf{C}_{\mathbf{p l}}$, giving

$$
\begin{aligned}
& C_{p u}=\frac{U S L-M}{U_{p}-M} \\
& C_{p l}=\frac{M-L S L}{M-L_{p}}
\end{aligned}
$$

$\mathrm{C}_{\mathrm{pk}}$ is defined as the minimum of $\mathrm{C}_{\mathrm{pu}}$ and $\mathrm{C}_{\mathrm{pl}}$ as follows:

$$
\mathrm{C}_{\mathrm{pk}}=\min \left\{\frac{\mathrm{USL}-\mathrm{M}}{\mathrm{U}_{\mathrm{p}}-\mathrm{M}}, \frac{\mathrm{M}-\mathrm{LSL}}{\mathrm{M}-\mathrm{L}_{\mathrm{p}}}\right\}
$$




\subsection{Procedure for Calculating PCIs by Clements' Method}

In the calculation of PCIs by Clements' method, estimates of the mean, standard deviation, skewness and kurtosis of the process data are required, and they should be calculated from a stable, in control process. USL and LSL are usually given externally by the design engineers according to the design requirements of the product.

The following steps give the procedure for calculating PCIs by Clements' Method.

1. LSL and USL are known values since they are determined by the design engineers.

2. The process mean and standard deviation are estimated by $\overline{\boldsymbol{X}}$ and S, respectively.

3. Skewness (sk) and kurtosis (kr) are estimated by the traditional method which is based on the third and the fourth central moments. (Detailed formulas are given in section 3)

4. Standardized values of $\mathbf{L}_{\mathbf{p}}, \mathbf{U}_{\mathbf{p}}$ and $\mathbf{M}$ (denoted by $\mathbf{L}_{\mathbf{p}}^{\prime}, \mathbf{U}_{\mathbf{p}}^{\prime}$ and $\mathbf{M}^{\prime}$ ) are obtained from the tables provided by [6].

5. $\mathbf{L}_{\mathbf{p}}, \mathbf{U}_{\mathbf{p}}$ and $\mathrm{M}$ are estimated as follows

$$
\mathbf{L}_{\mathbf{p}}=\overline{\mathbf{x}}-\mathbf{s} \mathbf{L}_{\mathbf{p}}^{\prime}, \quad \mathbf{U}_{\mathbf{p}}=\overline{\mathbf{x}}+\mathbf{s} \mathbf{U}_{\mathbf{p}}^{\prime} \quad, \quad \mathbf{M}=\overline{\mathbf{x}}+\mathbf{s} \mathbf{M}^{\prime}
$$

6. PCIs are calculated from equations (3)-(6).

As mentioned before, the given procedure for calculating PCIs employs traditional estimators of skewness and kurtosis. In this study, the use of more robust estimators of skewness and kurtosis in the calculation of PCIs are considered and an extensive simulation work is conducted for comparison. Skewness and kurtosis measures used in this study are explained in detail in section 3 .

\section{SKEWNESS AND KURTOSIS MEASURES}

Suppose that observations are obtained from a stable process and they are denoted by $\left\{\mathrm{y}_{\mathrm{i}}\right\}, \mathrm{i}=1,2, . ., \mathrm{n}$. Moreover, $\mathrm{y}_{\mathrm{i}}$ 's are identically, independently distributed and have the cumulative distribution function of $\mathrm{F}$, and $\mathrm{F}^{-1}$ stands for the inverse of the cumulative distribution function. The traditional coefficients of skewness and kurtosis for $\mathrm{y}_{\mathrm{i}}$ are given by

$$
\begin{aligned}
& \mathrm{sk}_{1}=\mathrm{E}\left(\frac{\mathrm{y}_{\mathrm{i}}-\mu}{\sigma}\right)^{3} \\
& \mathrm{kr}_{1}=\mathrm{E}\left(\frac{\mathrm{y}_{\mathrm{i}}-\mu}{\sigma}\right)^{4}-3
\end{aligned}
$$

where $\mu=\mathrm{E}\left(\mathrm{y}_{\mathrm{i}}\right)$ and $\sigma=\mathrm{E}\left(\mathrm{y}_{\mathrm{i}}-\mu\right)^{2}$. The following estimators are usually used in order to estimate $\mathrm{sk}_{1}$ and $\mathrm{kr}_{1}$.

$$
\begin{gathered}
\widehat{\mathrm{sk}}_{1}=\mathrm{n}^{-1} \sum_{\mathrm{i}=1}^{\mathrm{n}}\left(\frac{\mathrm{y}_{\mathrm{i}}-\widehat{\mu}}{\widehat{\sigma}}\right)^{3} \\
\widehat{\mathrm{kr}}_{1}=\mathrm{n}^{-1} \sum_{\mathrm{i}=1}^{\mathrm{n}}\left(\frac{\mathrm{y}_{\mathrm{i}}-\widehat{\mu}}{\widehat{\sigma}}\right)^{4}-3
\end{gathered}
$$

where $\hat{\mu}=\mathrm{n}^{-1} \sum_{\mathrm{i}=1}^{\mathrm{n}} \mathrm{y}_{\mathrm{i}}$ and $\widehat{\sigma}=\mathrm{n}^{-1} \sum_{\mathrm{i}=1}^{\mathrm{n}}\left(\mathrm{y}_{\mathrm{i}}-\hat{\mu}\right)^{2}$.

$\widehat{\mathrm{sk}}_{1}$ and $\widehat{\mathrm{kr}}_{1}$ become arbitrarily large when there are one or more large outliers in the data. Therefore, some other measures of skewness and kurtosis that are less sensitive to the outliers were proposed in the literature. For example, Bowley [20] proposed a coefficient of skewness based on quantiles

$$
\mathrm{sk}_{2}=\frac{\mathrm{Q}_{3}+\mathrm{Q}_{1}-2 \mathrm{Q}_{2}}{\mathrm{Q}_{3}-\mathrm{Q}_{1}}
$$


where $\mathrm{Q}_{\mathrm{k}}$ is the $\mathrm{k}^{\text {th }}$ quartile of $\mathrm{y}_{\mathrm{i}}$; that is, $\mathrm{Q}_{1}=\mathrm{F}^{-1}(0.25), \mathrm{Q}_{2}=\mathrm{F}^{-1}(0.5)$, and $\mathrm{Q}_{3}=\mathrm{F}^{-1}(0.75)$. The estimator of $\mathrm{sk}_{2}$ is given by

$$
\widehat{\mathrm{sk}}_{2}=\frac{\widehat{\mathrm{Q}}_{3}+\widehat{\mathrm{Q}}_{1}-2 \widehat{\mathrm{Q}}_{2}}{\widehat{\mathrm{Q}}_{3}-\widehat{\mathrm{Q}}_{1}}
$$

where $\widehat{\mathrm{Q}}_{\mathrm{k}}$ is the $\mathrm{k}^{\text {th }}$ sample quartile.

The Bowley coefficient of skewness has been generalized by Hinkley [21],

$$
\operatorname{sk}_{3}(\alpha)=\frac{\mathrm{F}^{-1}(1-\alpha)+\mathrm{F}^{-1}(\alpha)-2 \mathrm{Q}_{2}}{\mathrm{~F}^{-1}(1-\alpha)-\mathrm{F}^{-1}(\alpha)}
$$

for any $\alpha$ between 0 and 0.5 .

In order to remove the dependence of this measure on the value of $\alpha$, Groeneveld and Meeden [22] integrated out $\alpha$ as:

$$
\mathrm{sk}_{3}=\frac{\int_{0}^{0.5}\left\{\mathrm{~F}^{-1}(1-\alpha)+\mathrm{F}^{-1}(\alpha)-2 \mathrm{Q}_{2}\right\} \mathrm{d} \alpha}{\int_{0}^{0.5}\left\{\mathrm{~F}^{-1}(1-\alpha)-\mathrm{F}^{-1}(\alpha)\right\} \mathrm{d} \alpha}=\frac{\mu-\mathrm{Q}_{2}}{\mathrm{E}\left|\mathrm{y}_{\mathrm{t}}-\mathrm{Q}_{2}\right|}
$$

and this coefficient of skewness is estimated by

$$
\widehat{\mathrm{sk}}_{3}=\frac{\widehat{\mu}-\widehat{\mathrm{Q}}_{2}}{\mathrm{n}^{-1} \sum_{\mathrm{i}=1}^{\mathrm{n}}\left|\mathrm{y}_{\mathrm{i}}-\widehat{\mathrm{Q}}_{2}\right|}
$$

The final measure of skewness used in this study is the Pearson coefficient [23], and it was obtained by replacing the denominator in $\mathrm{sk}_{3}$ with the standard deviation.

$$
\mathrm{sk}_{4}=\frac{\mu-Q_{2}}{\sigma}
$$

The following is the estimator of $\mathrm{sk}_{4}$.

$$
\widehat{\mathrm{sk}}_{4}=\frac{\widehat{\mu}-\widehat{Q}_{2}}{\widehat{\sigma}}
$$

Moors [24] proposed an alternative measure of kurtosis which was given by

$$
\mathrm{kr}_{2}^{\prime}=\frac{\left(\mathrm{E}_{7}-\mathrm{E}_{5}\right)+\left(\mathrm{E}_{3}-\mathrm{E}_{1}\right)}{\mathrm{E}_{6}-\mathrm{E}_{2}}
$$

where $\mathrm{E}_{\mathrm{k}}$ is the $\mathrm{k}^{\text {th }}$ octile, i.e. $\mathrm{E}_{\mathrm{k}}=\mathrm{F}^{-1}(k / 8)$ for $\mathrm{k}=1,2, \ldots, 7$.

Moors coefficient of kurtosis is centered at the value for the standard normal distribution which is equal to 1.23 [19]. Therefore, the centered coefficient was given by

$$
\mathrm{kr}_{2}=\frac{\left(\mathrm{E}_{7}-\mathrm{E}_{5}\right)+\left(\mathrm{E}_{3}-\mathrm{E}_{1}\right)}{\mathrm{E}_{6}-\mathrm{E}_{2}}-1.23
$$

and the estimator of $\mathrm{kr}_{2}$ is

$$
\widehat{\mathrm{kr}}_{2}=\frac{\left(\widehat{\mathrm{E}}_{7}-\widehat{\mathrm{E}}_{5}\right)+\left(\widehat{\mathrm{E}}_{3}-\widehat{\mathrm{E}}_{1}\right)}{\widehat{\mathrm{E}}_{6}-\widehat{\mathrm{E}}_{2}}-1.23
$$

where $\widehat{\mathrm{E}}_{\mathrm{k}}$ is the $\mathrm{k}^{\mathrm{th}}$ sample octile.

Hogg $[25,26]$ suggested another measure of kurtosis which is given by $\frac{\mathrm{U}_{\alpha}-\mathrm{L}_{\alpha}}{\mathrm{U}_{\beta}-\mathrm{L}_{\beta}}$ where $\mathrm{U}_{\alpha}\left(\mathrm{L}_{\alpha}\right)$ is the average of the upper(lower) $\alpha$ quantiles defined as:

$\mathrm{U}_{\alpha}=\int_{1-\alpha}^{1} \mathrm{~F}^{-1}(\mathrm{y}) \mathrm{dy}, \quad \mathrm{L}_{\alpha}=\int_{0}^{\alpha} \mathrm{F}^{-1}(\mathrm{y}) \mathrm{dy}, \quad$ for $\alpha \in(0,1)$ 
According to Hogg's simulation experiments, $\alpha=0.05$ and $\beta=0.5$ gave the most satisfactory results. Adopting these values for $\alpha$ and $\beta$, Hogg coefficient was calculated as 2.59 for standard normal distribution [19]. Hence, the centered Hogg coefficient was given by

$$
\mathrm{kr}_{3}=\frac{\mathrm{U}_{0.05}-\mathrm{L}_{0.05}}{\mathrm{U}_{0.5}-\mathrm{L}_{0.5}}-2.59
$$

The estimator of $\mathrm{kr}_{3}$ is

$$
\widehat{\mathrm{kr}}_{3}=\frac{\widehat{\mathrm{U}}_{0.05}-\widehat{\mathrm{L}}_{0.05}}{\widehat{\mathrm{U}}_{0.5}-\widehat{\mathrm{L}}_{0.5}}-2.59
$$

where

$\widehat{\mathrm{U}}_{\alpha}=\frac{1}{\mathrm{n}_{\mathrm{u}}(\alpha)} \sum_{\mathrm{y}_{\mathrm{i}}>\widehat{\mathrm{P}}_{1-\alpha}} \mathrm{y}_{\mathrm{i}}, \quad \hat{\mathrm{L}}_{\alpha}=\frac{1}{\mathrm{n}_{\mathrm{l}}(\alpha)} \sum_{\mathrm{y}_{\mathrm{i}}<\widehat{\mathrm{P}}_{\alpha}} \mathrm{y}_{\mathrm{i}}, \quad$ for $\alpha \in(0,1)$

$\widehat{\mathrm{P}}_{\alpha}: \alpha^{\text {th }}$ sample percentile

$\mathrm{n}_{\mathrm{u}}(\alpha)$ : Number of cases satisfying the condition $\mathrm{y}_{\mathrm{i}}>\widehat{\mathrm{P}}_{1-\alpha}$

$\mathrm{n}_{\mathrm{l}}(\alpha)$ : Number of cases satisfying the condition $\mathrm{y}_{\mathrm{i}}<\widehat{\mathrm{P}}_{\alpha}$

The last measure of kurtosis, which is based on the quantiles was suggested by Crow and Siddiqui [18] and given as follows:

$\frac{F^{-1}(1-\alpha)-F^{-1}(\alpha)}{F^{-1}(1-\beta)-F^{-1}(\beta)} \quad$ for $\alpha, \beta \in(0,1)$.

Their suggestion for $\alpha$ and $\beta$ are 0.025 and 0.25 , respectively. For standard normal distribution the value of the coefficient was calculated as 2.91 [19] and the centered coefficient was given by

$$
\mathrm{kr}_{4}=\frac{\mathrm{F}^{-1}(0.975)-\mathrm{F}^{-1}(0.025)}{\mathrm{F}^{-1}(0.75)-\mathrm{F}^{-1}(0.25)}-2.91
$$

The following estimator is used for $\mathrm{kr}_{4}$.

$$
\widehat{\mathrm{kr}}_{4}=\frac{\widehat{\mathrm{P}}_{0.975}-\widehat{\mathrm{P}}_{0.025}}{\widehat{\mathrm{P}}_{0.75}-\widehat{\mathrm{P}}_{0.25}}-2.91
$$

where $\widehat{\mathrm{P}}_{\alpha}$ is the $\alpha^{\text {th }}$ sample percentile.

\section{SIMULATION STUDY}

In the simulation study, one sided tolerance limit for PCI is used and $\mathrm{C}_{\mathrm{pu}}$ is computed as a comparison measure. In Rivera et. al.[27], USLs of the non-normal distributions are determined in such a way that fraction of non-conforming parts will give the same fraction of non-conforming parts under normality that corresponds to target PCI value. In this study, similar approach is used to compute USL corresponding to the target $\mathrm{C}_{\mathrm{pu}}$. As in Hosseinifard et.al. [14], USL is obtained by equation (25).

$$
\mathbf{U S L}=\mathbf{F}^{-1}\left(\boldsymbol{\Phi}\left(3 \mathrm{C}_{\mathrm{pu}}\right)\right)
$$

where $\mathrm{F}$ is the cumulative distribution function of the non-normal data and $\boldsymbol{\Phi}$ is the cumulative normal density function.

Target $C_{p u}$ values are taken as $0.5,1.0$, and 1.5. Weibull and lognormal distributions are considered and $\mathrm{C}_{\mathrm{pu}}$ values are estimated in order to compare with the target values. Estimation of $\mathrm{C}_{\mathrm{pu}}$ is based on 10000 samples of size $n=100$. For comparative purposes, MSEs are computed and presented in Table 1 and Table 2 when different skewness and kurtosis estimators are used in the implementation of Clements' method. Table 1 and Table 2 are representing the simulation results for the Weibull distribution and log- 
normal distribution, respectively, with different parameter values. In these tables minimum MSEs are given in bold. In order to carry out these simulations a program was written in Matlab.

Table1. MSE of the estimator of $\mathrm{C}_{\mathrm{pu}}$ for Weibull distribution with different shape parameters when different pairs of skewness and kurtosis estimators are used in the estimation of $\mathrm{C}_{\mathrm{pu}}$ by Clements' method.

\begin{tabular}{|c|c|c|c|c|c|c|}
\hline \multicolumn{3}{|c|}{ Cpu } & $\widehat{k r}_{1}$ & $\widehat{k r}_{2}$ & $\widehat{k r}_{3}$ & $\widehat{k r}_{4}$ \\
\hline \multirow{12}{*}{$\begin{array}{c}\text { wbl }(\text { scale }=1, \text { shape }=3) \\
\text { sk }=0.1681 \quad k r=-0.2705\end{array}$} & \multirow[t]{4}{*}{0.5} & \multirow{4}{*}{$\begin{array}{l}\widehat{s k}_{1} \\
\widehat{s k \boldsymbol{k}}_{2} \\
\widehat{s k}_{3} \\
\widehat{s k}_{4}\end{array}$} & 0.01367 & 0.00463 & 0.00560 & 0.00848 \\
\hline & & & 0.01638 & 0.00481 & 0.00625 & 0.00991 \\
\hline & & & 0.01430 & 0.00386 & 0.00499 & 0.00859 \\
\hline & & & 0.01364 & 0.00370 & 0.00480 & 0.00829 \\
\hline & \multirow[t]{4}{*}{1} & \multirow{4}{*}{$\begin{array}{l}\widehat{s k}_{1} \\
\widehat{s k}_{2} \\
\widehat{s k}_{3} \\
\widehat{s k}_{4}\end{array}$} & 0.04777 & 0.01429 & 0.01610 & 0.02839 \\
\hline & & & 0.05561 & 0.01377 & 0.01897 & 0.03239 \\
\hline & & & 0.04625 & 0.01115 & 0.01398 & 0.02724 \\
\hline & & & 0.04526 & $\mathbf{0 . 0 1 0 0 5}$ & 0.01288 & 0.02658 \\
\hline & \multirow[t]{4}{*}{1.5} & \multirow{4}{*}{$\begin{array}{l}\widehat{s k}_{1} \\
\widehat{s k}_{2} \\
\widehat{s k}_{3} \\
\widehat{s k}_{4}\end{array}$} & 0.09074 & 0.03205 & 0.03336 & 0.05688 \\
\hline & & & 0.10032 & 0.02554 & 0.03218 & 0.05849 \\
\hline & & & 0.08248 & 0.02121 & 0.02306 & 0.04811 \\
\hline & & & 0.08212 & 0.01920 & 0.02133 & 0.04516 \\
\hline \multirow{12}{*}{$\begin{array}{c}\text { wbl }(\text { scale }=1, \text { shape }=2) \\
\text { sk }=0.6311 \quad k r=0.2451\end{array}$} & \multirow[t]{4}{*}{0.5} & \multirow{4}{*}{$\begin{array}{l}\widehat{s k}_{1} \\
\widehat{s k}_{2} \\
\widehat{s k}_{3} \\
\widehat{s k}_{4}\end{array}$} & 0.01257 & 0.01094 & 0.01772 & 0.03313 \\
\hline & & & 0.01565 & 0.00761 & 0.00990 & 0.01463 \\
\hline & & & 0.01340 & 0.00587 & 0.00761 & 0.01175 \\
\hline & & & 0.01327 & 0.00591 & 0.00771 & 0.01257 \\
\hline & \multirow[t]{4}{*}{1} & \multirow{4}{*}{$\begin{array}{l}\widehat{s k}_{1} \\
\widehat{s k}_{2} \\
\widehat{s k}_{3} \\
\widehat{s k}_{4}\end{array}$} & 0.07126 & 0.17374 & 0.25984 & 0.13685 \\
\hline & & & 0.11450 & 0.06544 & 0.08599 & 0.11582 \\
\hline & & & 0.09667 & 0.04915 & 0.06610 & 0.09368 \\
\hline & & & 0.09387 & 0.05156 & 0.06968 & 0.10018 \\
\hline & \multirow[t]{4}{*}{1.5} & \multirow{4}{*}{$\begin{array}{l}\widehat{s k}_{1} \\
\widehat{s k}_{2} \\
\widehat{s k}_{3} \\
\widehat{s k}_{4}\end{array}$} & 0.22558 & 0.42159 & 0.36326 & 0.58543 \\
\hline & & & 0.35355 & 0.21913 & 0.28607 & 0.37376 \\
\hline & & & 0.29608 & 0.17496 & 0.22567 & 0.29965 \\
\hline & & & 0.30450 & 0.18332 & 0.23408 & 0.32226 \\
\hline \multirow{12}{*}{$\begin{array}{c}\text { wbl(scale=1, shape=1.5) } \\
\text { sk }=1.0720 \quad k r=1.3904\end{array}$} & \multirow[t]{4}{*}{0.5} & \multirow{4}{*}{$\begin{array}{l}\boldsymbol{s k}_{1} \\
\widehat{s k}_{2} \\
\widehat{s k}_{3} \\
\widehat{s k}_{4}\end{array}$} & 0.01874 & $*$ & $*$ & * \\
\hline & & & 0.01644 & 0.01003 & 0.01222 & 0.01686 \\
\hline & & & 0.01211 & 0.00765 & 0.00910 & 0.01345 \\
\hline & & & 0.01174 & 0.00822 & 0.00982 & 0.01485 \\
\hline & \multirow[t]{4}{*}{1} & \multirow{4}{*}{$\begin{array}{l}\widehat{s k}_{1} \\
\widehat{s k}_{2} \\
\widehat{s k}_{3} \\
\widehat{s k}_{4}\end{array}$} & 0.10922 & $*$ & * & * \\
\hline & & & 0.13769 & 0.19074 & 0.22086 & 0.25982 \\
\hline & & & 0.12543 & 0.14987 & 0.17429 & 0.22243 \\
\hline & & & 0.12849 & 0.16295 & 0.18806 & 0.22984 \\
\hline & \multirow[t]{4}{*}{1.5} & $\widehat{s k}_{1}$ & 0.55984 & $*$ & $*$ & $*$ \\
\hline & & $\widehat{s k}_{2}$ & 0.65436 & 0.91485 & 1.02458 & 1.17467 \\
\hline & & $\widehat{s \boldsymbol{k}}_{3}$ & 0.56473 & 0.76900 & 0.85416 & 1.01083 \\
\hline & & $\widehat{s k}_{4}$ & 0.59813 & 0.82026 & 0.90437 & 1.04805 \\
\hline
\end{tabular}

* : represents very large number

According to Table 1, for small values of skewness and kurtosis, using traditional estimators $\left(\widehat{\mathbf{s k}}_{\mathbf{1}}-\widehat{\mathbf{k r}}_{\mathbf{1}}\right)$ in the estimation of $\mathrm{C}_{\mathrm{pu}}$ results in larger MSE as compared to the use of other estimators of skewness and kurtosis. Especially, using $\widehat{\mathbf{s k}}_{3}$ or $\widehat{\mathbf{s k}}_{\mathbf{4}}$ in combination with $\widehat{\mathbf{k r}}_{\mathbf{2}}$ in the estimation of $\mathrm{C}_{\mathrm{pu}}$ by Clements' method might be preferred by the practitioners since their corresponding MSE is considerably less than the MSE of $\hat{\mathbf{C}}_{\mathbf{p u}}$ which is obtained by traditional estimators.

Table2. MSE of the estimator of $\mathrm{C}_{\mathrm{pu}}$ for lognormal distribution with different shape parameters when different pairs of skewness and kurtosis estimators are used in the estimation of $\mathrm{C}_{\mathrm{pu}}$ by Clements' method. 
Aytaçoğlu and Genç / Eskişehir Tech. Univ. J. of Sci. and Technology A - Appl. Sci. and Eng. 20 (4) - 2019

\begin{tabular}{|c|c|c|c|c|c|c|}
\hline \multicolumn{3}{|c|}{ Cpu } & $\widehat{k r}_{1}$ & $\widehat{k r}_{2}$ & $\widehat{k r}_{3}$ & $\widehat{k r}_{4}$ \\
\hline \multirow{12}{*}{$\begin{array}{c}\log n(\mu=0, \sigma=0.05) \\
\text { sk }=0.1502 \quad k r=0.0401\end{array}$} & \multirow[t]{4}{*}{0.5} & \multirow{4}{*}{$\begin{array}{l}\widehat{s k}_{1} \\
\widehat{s k}_{2} \\
\widehat{s k}_{3} \\
\widehat{s k}_{4}\end{array}$} & 0.01147 & 0.00657 & 0.00696 & 0.00922 \\
\hline & & & 0.01008 & 0.00458 & 0.00491 & 0.00761 \\
\hline & & & 0.00869 & 0.00400 & 0.00437 & 0.00651 \\
\hline & & & 0.00825 & 0.00376 & 0.00412 & 0.00639 \\
\hline & \multirow[t]{4}{*}{1} & \multirow{4}{*}{$\begin{array}{l}\widehat{s \boldsymbol{k}}_{1} \\
\widehat{\boldsymbol{s k}}_{2} \\
\widehat{\boldsymbol{s k}}_{3} \\
\widehat{\boldsymbol{s k}}_{4}\end{array}$} & 0.05194 & 0.02982 & 0.02930 & 0.04254 \\
\hline & & & 0.04835 & 0.02234 & 0.02565 & 0.03728 \\
\hline & & & 0.04316 & 0.01758 & 0.02007 & 0.03042 \\
\hline & & & 0.04161 & 0.01675 & 0.01959 & 0.03072 \\
\hline & \multirow[t]{4}{*}{1.5} & \multirow{4}{*}{$\begin{array}{l}\widehat{s k}_{1} \\
\widehat{s k}_{2} \\
\widehat{s k}_{3} \\
\widehat{s k}_{4}\end{array}$} & 0.15072 & 0.07758 & 0.09156 & 0.11947 \\
\hline & & & 0.15308 & 0.07619 & 0.08393 & 0.11682 \\
\hline & & & 0.12955 & 0.06264 & 0.06938 & 0.09911 \\
\hline & & & 0.12515 & 0.05946 & 0.06873 & 0.09347 \\
\hline \multirow{12}{*}{$\begin{array}{c}\operatorname{logn}(\mu=0, \sigma=0.10) \\
\text { sk }=0.3018 \quad k r=0.1623\end{array}$} & \multirow[t]{4}{*}{0.5} & \multirow{4}{*}{$\begin{array}{l}\widehat{s k}_{1} \\
\widehat{s k}_{2} \\
\widehat{s k}_{3} \\
\widehat{s k}_{4}\end{array}$} & 0.01176 & 0.00609 & 0.00711 & 0.00878 \\
\hline & & & 0.01034 & 0.00510 & 0.00582 & 0.00834 \\
\hline & & & 0.00949 & 0.00455 & 0.00487 & 0.00705 \\
\hline & & & 0.00928 & 0.00441 & 0.00471 & 0.00720 \\
\hline & \multirow[t]{4}{*}{1} & \multirow{4}{*}{$\begin{array}{l}\widehat{s k}_{1} \\
\widehat{s k}_{2} \\
\widehat{s k}_{3} \\
\widehat{s k}_{4}\end{array}$} & 0.05992 & 0.15722 & 0.03642 & 0.16736 \\
\hline & & & 0.07135 & 0.04122 & 0.04437 & 0.05788 \\
\hline & & & 0.06320 & 0.03246 & 0.03645 & 0.04850 \\
\hline & & & 0.06066 & 0.03203 & 0.03637 & 0.04821 \\
\hline & \multirow[t]{4}{*}{1.5} & \multirow{4}{*}{$\begin{array}{l}\widehat{s k}_{1} \\
\widehat{s k}_{2} \\
\widehat{s k}_{3} \\
\widehat{s k}_{4}\end{array}$} & 0.22658 & 0.35713 & 0.14682 & 0.29173 \\
\hline & & & 0.28444 & 0.18628 & 0.20959 & 0.24481 \\
\hline & & & 0.25196 & 0.16206 & 0.17658 & 0.20935 \\
\hline & & & 0.25053 & 0.15857 & 0.17863 & 0.21191 \\
\hline \multirow{12}{*}{$\begin{array}{c}\operatorname{logn}(\mu=0, \sigma=0.40) \\
\text { sk }=1.3219 \quad \mathrm{kr}=3.26\end{array}$} & \multirow[t]{4}{*}{0.5} & \multirow{4}{*}{$\begin{array}{l}\widehat{\boldsymbol{s k}}_{1} \\
\widehat{\boldsymbol{s k}}_{2} \\
\widehat{\boldsymbol{s k}}_{3} \\
\widehat{s k}_{4}\end{array}$} & 0.02719 & $*$ & * & * \\
\hline & & & 0.01466 & 0.00984 & 0.01022 & 0.01201 \\
\hline & & & 0.02935 & 0.00847 & 0.00927 & 0.01155 \\
\hline & & & 0.01634 & 0.00844 & 0.00949 & 0.01132 \\
\hline & \multirow[t]{4}{*}{1} & \multirow{4}{*}{$\begin{array}{l}\widehat{s k}_{1} \\
\widehat{s k}_{2} \\
\widehat{s k}_{3} \\
\widehat{s k}_{4}\end{array}$} & 0.17716 & $*$ & $*$ & $*$ \\
\hline & & & 0.28336 & 0.42216 & 0.41290 & 0.41566 \\
\hline & & & 0.27426 & 0.36440 & 0.34663 & 0.35257 \\
\hline & & & 0.22575 & 0.38488 & 0.36941 & 0.37907 \\
\hline & \multirow[t]{4}{*}{1.5} & \multirow{4}{*}{$\begin{array}{l}\widehat{\boldsymbol{s k}}_{1} \\
\widehat{\boldsymbol{s k}}_{2} \\
\widehat{\boldsymbol{s k}}_{3} \\
\widehat{\boldsymbol{s k}}_{1}\end{array}$} & 2.37022 & $*$ & $*$ & $*$ \\
\hline & & & 3.29255 & 4.60860 & 4.44679 & 4.40649 \\
\hline & & & 2.75485 & 4.08871 & 3.97120 & 3.95354 \\
\hline & & & 3.98982 & 4.29578 & 4.16337 & 4.09141 \\
\hline
\end{tabular}

As the skewness and kurtosis of the process data increase, using traditional estimators of them results in smaller MSE of $\widehat{\mathbf{C}}_{\mathbf{p u}}$. Unless $C_{\mathrm{pu}}$ is very small, it is better to use traditional estimators $\left(\widehat{\mathbf{s k}}_{\mathbf{1}}-\widehat{\mathbf{k r}}_{\mathbf{1}}\right)$ in the estimation of $\mathrm{C}_{\mathrm{pu}}$.

For large values of skewness and kurtosis, the use of $\widehat{\mathbf{k r}}_{2}, \widehat{\mathbf{k r}}_{3}$ and $\widehat{\mathbf{k r}}_{\mathbf{4}}$ with $\widehat{\mathbf{s k}}_{\mathbf{1}}$ gives very large MSE values of $\widehat{\mathbf{C}}_{\mathbf{p u}}$. Therefore, $\widehat{\mathbf{s k}}_{\mathbf{1}}-\widehat{\mathbf{k r}}_{2}, \widehat{\mathbf{s k}}_{\mathbf{1}}-\widehat{\mathbf{k r}}_{3}$ and $\widehat{\mathbf{s k}}_{\mathbf{1}}-\widehat{\mathbf{k r}}_{\mathbf{4}}$ pairs should not be preferred in the estimation of $\mathrm{C}_{\mathrm{pu}}$.

When we examine Table 2, for small values of skewness and kurtosis, generally using $\widehat{\mathbf{s k}}_{\mathbf{4}}$ and $\widehat{\mathbf{k r}}_{\mathbf{2}}$ pair in the estimation of $\mathrm{C}_{\mathrm{pu}}$ by Clement's method results in smaller MSE as compared to the use of other estimators of skewness and kurtosis. As skewness and kurtosis of the data get larger MSE values become very large, especially when $\widehat{\mathbf{s k}}_{\mathbf{1}}-\widehat{\mathbf{k r}}_{\mathbf{2}}, \widehat{\mathbf{s k}}_{\mathbf{1}}-\widehat{\mathbf{k r}}_{3}$, and $\widehat{\mathbf{s k}}_{\mathbf{1}}-\widehat{\mathbf{k r}}_{\mathbf{4}}$ pairs are used in the estimation of $\mathrm{C}_{\mathrm{pu}}$. Therefore, if the data has large skewness and kurtosis, instead of these pairs, traditional estimators of 
skewness and kurtosis, $\widehat{\mathbf{s k}}_{\mathbf{1}}$ and $\widehat{\mathbf{k r}}_{\mathbf{1}}$, should be preferred for estimation of $\mathrm{C}_{\mathrm{pu}}$. However, if the skewness and kurtosis of the data are not so large one can prefer to use $\widehat{\mathbf{s k}}_{\mathbf{4}}-\widehat{\mathbf{k r}}_{2}$ pair in the estimation of $\mathrm{C}_{\mathrm{pu}}$ by Clement's method.

\section{A REAL LIFE APPLICATION TO OIL PUMP MANUFACTURING}

In an engine oil pump, there are several important quality characteristics that have to conform to product specifications determined by the design engineers so that the oil pump performs as expected. It is quite significant that those quality characteristics are individually monitored to their tolerance limits and effects of tolerance stack-up due to those limits, which can simply be expressed as the accumulation of dimensional errors, are kept under control. Highly accurate and precise CMMs (Coordinate Measuring Machine) are used to measure those quality characteristics knowing that any dimensional nonconformity may negatively influence product performance or even lead to total product failure causing drastic car engine break. Measurement results are then used to perform process capability inspections to ensure that the process is under control. A correct evaluation of process capability requires proper analysis of the available data, determination of the type of statistical distribution and then computation of the process capability indices. Distributions of the most of the quality characteristics in oil pump manufacturing process do not fit to normal distribution contrary to expectations. Therefore, standard PCI's may cause misleading interpretations.

This study aims investigation and interpretation of machining process capability for body part pocket bottom surface flatness which has considerable effect on product performance. Any undetected nonconformity of this characteristic has major potential consequences such as insufficient engine cooling and thus low engine performance, rapid wear on engine components thus shorter engine life and in the worst case, complete engine burn out during end customer use.

In the present application, tolerance limit of the pocket bottom surface flatness was defined as 30 microns by the technical drawing. Measurements were carried out on one single CMM. Parts were located on fixtures using three support surfaces of the body part. After measurement datums were set by CMM, 1500 different coordinate measurements were made for each part from pre-defined scattered positions representing the whole surface as a point cloud. By the help of those measurements, a virtual flat surface was fit by CMM software using Least-Squares Fitting Algorithms based on orthogonal distance regression. The orthogonal distance between the upper and lower peak points of the point cloud along the normal of the fit flat surface gave the flatness value.

Totally 233 pcs were inspected in this study in order to correctly calculate the capability indices and thus make proper interpretation of the whole flatness data.

Initially the histogram and normal probability plot for the flatness data are obtained and given in Figure 1.

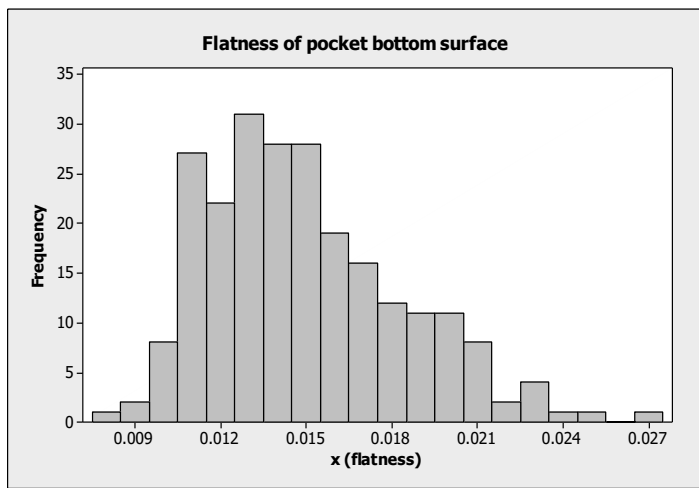

(a)

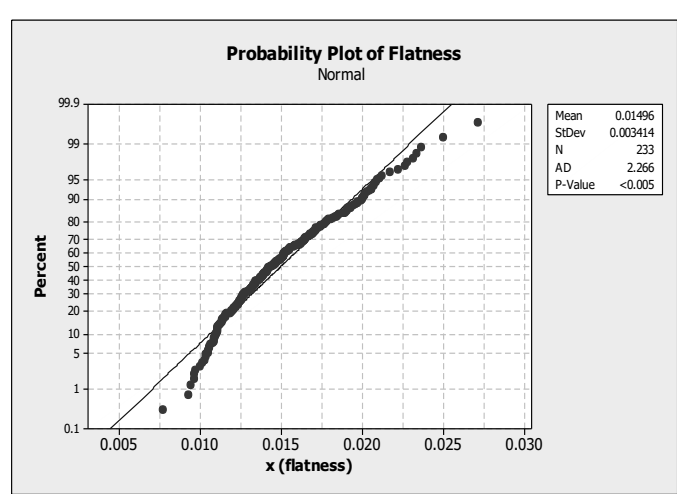

(b)

Figure 1. (a) Histogram of pocket bottom flatness data (b) Normal probability plot of pocket bottom flatness data 
Aytaçoğlu and Genç / Eskişehir Tech. Univ. J. of Sci. and Technology A - Appl. Sci. and Eng. 20 (4) - 2019

When the histogram and the normal probability plot are examined one can easily conclude that the flatness data is not distributed as Normal ( $\mathrm{p}$ value < 0.005). Therefore, it is not appropriate to use standard PCIs in the capability analysis and the application of Clements' method with the possible different estimators of skewness and kurtosis given in section 3 is presented in this section.

The procedure for calculating PCIs by Clements' method given in section 2 is conducted for the pocket bottom flatness data.

1. Note that the LSL and USL were 0.00 and $0.03 \mathrm{~mm}$, respectively. However, since flatness can not take a negative value, this process characteristic can be considered as having just USL $=$ $0.03 \mathrm{~mm}$.

2. The process mean and the standard deviation are estimated.

$\bar{x}=0.014962, \mathrm{~s}=0.003414$

3. Skewness and kurtosis of the flatness data are estimated by both traditional estimators and by the other robust estimators given in section 3. The obtained estimates are given in Table 3.

Table 3. Skewness and kurtosis estimates of pocket bottom flatness data.

\begin{tabular}{|l|l|l|l|}
\hline$\widehat{\boldsymbol{s k}}_{\mathbf{1}}$ & 0.6832 & $\widehat{\boldsymbol{k r}}_{\mathbf{1}}$ & 0.1794 \\
\hline$\widehat{\boldsymbol{s k}}_{\mathbf{2}}$ & 0.1460 & $\widehat{\boldsymbol{k r}}_{\mathbf{2}}$ & 0.0903 \\
\hline$\widehat{\boldsymbol{s k}}_{\mathbf{3}}$ & 0.2096 & $\widehat{\boldsymbol{k r}}_{\mathbf{3}}$ & -0.1704 \\
\hline$\widehat{\boldsymbol{s k}}_{\mathbf{4}}$ & 0.1663 & $\widehat{\boldsymbol{k r}}_{\mathbf{4}}$ & -0.1314 \\
\hline
\end{tabular}

4. Standardized values of $\mathrm{L}_{\mathrm{p}}, \mathrm{U}_{\mathrm{p}}$ and $\mathrm{M}$ (denoted by $\mathrm{L}_{\mathrm{p}}^{\prime}, \mathrm{U}_{\mathrm{p}}^{\prime}$ and $\mathrm{M}^{\prime}$ ) are obtained from the tables in [6] and given in Table 4.

Table 4. Standardized values of $L_{p}, U_{p}$ and $M$ obtained for different estimates of skewness and kurtosis of pocket bottom flatness data.

\begin{tabular}{|l|c|c|c|c|c|}
\cline { 3 - 6 } \multicolumn{1}{c|}{} & $\widehat{\boldsymbol{k r}}_{\mathbf{1}}=\mathbf{0 . 1 7 9 4}$ & $\widehat{\boldsymbol{k r}}_{\mathbf{2}}=\mathbf{0 . 0 9 0 3}$ & $\widehat{\boldsymbol{k r}}_{\mathbf{3}}=\mathbf{- 0 . 1 7 0 4}$ & $\widehat{\boldsymbol{k r}}_{\mathbf{4}}=\mathbf{- 0 . 1 3 1 4}$ \\
\hline \multirow{3}{*}{$\widehat{\boldsymbol{s k}}_{\mathbf{1}}=\mathbf{0 . 6 8 3 2}$} & $\boldsymbol{L}_{\boldsymbol{p}}^{\prime}$ & 1.7526 & 1.6858 & 1.4929 & 1.5216 \\
& $\boldsymbol{U}_{\boldsymbol{p}}^{\prime}$ & 3.5287 & 3.4408 & 3.1541 & 3.1993 \\
& $\boldsymbol{M}^{\prime}$ & 0.1689 & 0.1620 & 0.1790 & 0.1831 \\
\hline \multirow{3}{*}{$\widehat{\boldsymbol{s k}}_{\mathbf{2}}=\mathbf{0 . 1 4 6 0}$} & $\boldsymbol{L}_{\boldsymbol{p}}^{\prime}$ & 2.9020 & 2.8410 & 2.6383 & 2.6705 \\
& $\boldsymbol{U}_{\boldsymbol{p}}^{\prime}$ & 3.2941 & 3.2322 & 3.0307 & 3.0624 \\
& $\boldsymbol{M}^{\prime}$ & 0.0266 & 0.0257 & 0.0270 & 0.0274 \\
\hline \multirow{3}{*}{$\widehat{\boldsymbol{s k}}_{\mathbf{3}}=\mathbf{0 . 2 0 9 6}$} & $\boldsymbol{L}_{\boldsymbol{p}}^{\prime}$ & 2.8016 & 2.7367 & 2.5282 & 2.5609 \\
& $\boldsymbol{U}_{\boldsymbol{p}}^{\prime}$ & 3.3575 & 3.2950 & 3.0914 & 3.1235 \\
& $\boldsymbol{M}^{\prime}$ & 0.0378 & 0.0368 & 0.0395 & 0.0401 \\
\hline \multirow{3}{*}{$\widehat{\boldsymbol{s k}}_{\mathbf{4}}=\mathbf{0 . 1 6 6 3}$} & $\boldsymbol{L}_{\boldsymbol{p}}^{\prime}$ & 2.8708 & 2.8085 & 2.6039 & 2.6362 \\
& $\boldsymbol{U}_{\boldsymbol{p}}^{\prime}$ & 3.3149 & 3.2529 & 3.0508 & 3.0827 \\
& $\boldsymbol{M}^{\prime}$ & 0.0301 & 0.0292 & 0.0309 & 0.0314 \\
\hline
\end{tabular}

5. $\mathbf{L}_{\mathbf{p}}, \mathbf{U}_{\mathbf{p}}$ and $\mathrm{M}$ are estimated as described in section 2 and they are given in Table 5 . 
Table 5. Calculated values of $L_{p}, U_{p}$ and $M$ for pocket bottom flatness data.

\begin{tabular}{|c|c|c|c|c|c|}
\cline { 3 - 6 } \multicolumn{2}{c|}{} & $\widehat{\boldsymbol{k r}}_{\mathbf{1}}=\mathbf{0 . 1 7 9 4}$ & $\widehat{\boldsymbol{k r}}_{\mathbf{2}}=\mathbf{0 . 0 9 0 3}$ & $\widehat{\boldsymbol{k r}}_{\mathbf{3}}=\mathbf{- 0 . 1 7 0 4}$ & $\widehat{\boldsymbol{k r}}_{\mathbf{4}}=\mathbf{- 0 . 1 3 1 4}$ \\
\hline \multirow{3}{*}{$\widehat{\boldsymbol{s k}}_{\mathbf{1}}=\mathbf{0 . 6 8 3 2}$} & $\boldsymbol{L}_{\boldsymbol{p}}$ & 0.0090 & 0.0092 & 0.0099 & 0.0098 \\
& $\boldsymbol{U}_{\boldsymbol{p}}$ & 0.0270 & 0.0267 & 0.0257 & 0.0259 \\
& $\boldsymbol{M}$ & 0.0155 & 0.0155 & 0.0156 & 0.0156 \\
\hline \multirow{3}{*}{$\widehat{\boldsymbol{s k}}_{\mathbf{2}}=\mathbf{0 . 1 4 6 0}$} & $\boldsymbol{L}_{\boldsymbol{p}}$ & 0.0051 & 0.0053 & 0.0060 & 0.0058 \\
& $\boldsymbol{U}_{\boldsymbol{p}}$ & 0.0262 & 0.0260 & 0.0253 & 0.0254 \\
& $\boldsymbol{M}$ & 0.0151 & 0.0150 & 0.0151 & 0.0151 \\
\hline \multirow{3}{*}{$\widehat{\boldsymbol{s k}}_{\mathbf{3}}=\mathbf{0 . 2 0 9 6}$} & $\boldsymbol{L}_{\boldsymbol{p}}$ & 0.0054 & 0.0056 & 0.0063 & 0.0062 \\
& $\boldsymbol{U}_{\boldsymbol{p}}$ & 0.0264 & 0.0262 & 0.0255 & 0.0256 \\
& $\boldsymbol{M}$ & 0.0151 & 0.0151 & 0.0151 & 0.0151 \\
\hline \multirow{3}{*}{$\widehat{\boldsymbol{s k}}_{\mathbf{4}}=\mathbf{0 . 1 6 6 3}$} & $\boldsymbol{L}_{\boldsymbol{p}}$ & 0.0052 & 0.0054 & 0.0061 & 0.0060 \\
& $\boldsymbol{U}_{\boldsymbol{p}}$ & 0.0263 & 0.0261 & 0.0254 & 0.0255 \\
& $\boldsymbol{M}$ & 0.0151 & 0.0151 & 0.0151 & 0.0151 \\
\hline
\end{tabular}

6. For the flatness data there is just USL given by the design engineers. Therefore, $\mathrm{C}_{\mathrm{pu}}$ values are estimated by using equation (4) in order to assess the capability of the process and they are given in Table 6.

Table 6. Estimated $\mathrm{C}_{\mathrm{pu}}$ values for pocket bottom flatness data.

\begin{tabular}{|l|c|c|c|c|}
\cline { 2 - 5 } \multicolumn{1}{c|}{} & $\widehat{\boldsymbol{k r}}_{\mathbf{1}}=\mathbf{0 . 1 7 9 4}$ & $\widehat{\boldsymbol{k r}}_{\mathbf{2}}=\mathbf{0 . 0 9 0 3}$ & $\widehat{\boldsymbol{k r}}_{\mathbf{3}}=\mathbf{- \mathbf { 0 . 1 7 0 4 }}$ & $\widehat{\boldsymbol{k r}}_{\mathbf{4}}=\mathbf{- \mathbf { 0 . 1 3 1 4 }}$ \\
\hline$\widehat{\boldsymbol{s k}}_{\mathbf{1}}=\mathbf{0 . 6 8 3 2}$ & 1.26 & 1.29 & 1.42 & 1.40 \\
$\widehat{\mathbf{s k}}_{2}=\mathbf{0 . 1 4 6 0}$ & 1.34 & 1.37 & 1.46 & 1.44 \\
$\widehat{\boldsymbol{s k}}_{3}=\mathbf{0 . 2 0 9 6}$ & 1.32 & $\mathbf{1 . 3 4}$ & 1.43 & 1.42 \\
$\widehat{\boldsymbol{s k}}_{\mathbf{4}}=\mathbf{0 . 1 6 6 3}$ & 1.33 & $\mathbf{1 . 3 6}$ & 1.45 & 1.43 \\
\hline
\end{tabular}

In Table $6, \mathrm{C}_{\mathrm{pu}}$ is estimated by using all possible estimators of skewness and kurtosis given in this study. When the histogram and the estimates of skewness and kurtosis of pocket bottom flatness data are examined, we see that the skewness of the flatness data is not so large and the kurtosis estimates fluctuate around 0 . In the previous section, simulation results showed us that if the skewness and kurtosis are not so large, instead of using traditional estimators of them it is better to use $\widehat{s k}_{3}-\widehat{k r}_{2}$ or $\widehat{s k}_{4}-\widehat{k r}_{2}$ pair in the estimation of $\mathrm{C}_{\mathrm{pu}}$. Therefore, $\mathrm{C}_{\mathrm{pu}}$ values that are estimated by using these pairs are given in bold in Table 6. Assessing the process capability with the $\mathrm{C}_{\mathrm{pu}}$ values estimated by the use of the traditional estimators $\left(\widehat{s k}_{1}-\widehat{k r}_{1}\right)$ may mislead the practitioner about the capability of the process. $\mathrm{C}_{\mathrm{pu}}$ for the flatness data which is estimated by the traditional estimators of skewness and kurtosis is smaller than the $\mathrm{C}_{\mathrm{pu}}$ estimated by using $\widehat{s k}_{3}-\widehat{k r}_{2}$ or $\widehat{s k}_{4}-\widehat{k r}_{2}$ pair, and smaller $\mathrm{C}_{\mathrm{pu}}$ is the indication of less capable process. In that case a practitioner may take an action although it is not actually needed.

\section{CONCLUSION}

When probability distribution of a process characteristic is non-normal, PCIs calculated using the traditional methods often causes misleading interpretation of process capability. Several methods were proposed in the literature in order to analyze the capability of process when the process characteristic is non-normal. One of the most widely discussed methods to handle non-normality is Clements' method. In this study, Clements' method was modified by changing the estimators of skewness and kurtosis used in the estimation procedure of PCIs. Instead of using traditional estimators of skewness and kurtosis, more robust estimators proposed in the literature were used in the estimation of PCIs by Clements' method. For comparison purposes, simulation study was conducted and results were obtained when a process characteristic has Weibull or log-normal distribution. Simulation results showed that if the 
Aytaçoğlu and Genç / Eskişehir Tech. Univ. J. of Sci. and Technology A - Appl. Sci. and Eng. 20 (4) - 2019

distribution of the process characteristic is not so much skewed and its kurtosis is not large then instead of using the traditional estimators of skewness and kurtosis it is better to use $\widehat{s k}_{3}-\widehat{k r}_{2}$ or $\widehat{s k}_{4}-\widehat{k r}_{2}$ pair in the estimation of $\mathrm{C}_{\mathrm{pu}}$ by Clements' method. A real life example regarding an oil pump manufacturing process was provided in section 5, and pocket bottom flatness characteristic in the engine oil pump was analyzed by estimating the $\mathrm{C}_{\mathrm{pu}}$ index in order to make interpretations about the capability of the process. Several $\mathrm{C}_{\mathrm{pu}}$ values were estimated by using different estimators of skewness and kurtosis. However, the use of $\widehat{\mathrm{C}}_{\mathrm{pu}}$ which utilizes $\widehat{s k}_{3}-\widehat{k r}_{2}$ or $\widehat{s k}_{4}-\widehat{k r}_{2}$ pair in the estimation procedure was suggested relying on the simulation results presented in section 4.

\section{REFERENCES}

[1] Box GEP, Cox DR. An analysis of transformations. J Roy Statist Soc B 1964; 26: 211-243.

[2] Johnson NL, Kotz S. Discrete Distributions. New York, NY, USA: John Wiley, 1969.

[3] Ryan TP. Statistical Methods for Quality Improvement. New York, NY, USA: John Wiley, 1989.

[4] Ryan TP, Schwertman NC. Optimal limits for attributes control charts. J Qual Technol 1997; 29: 86-96.

[5] Niaki STA, Abbasi B. Skewness reduction approach in multi-attribute process monitoring. Commun Stat - Theory Methods 2007; 36:12, 2313-2325.

[6] Clements JA. Process capability indices for non-normal calculations. Qual Prog 1989; 22: 49-55.

[7] Liu PH, Chen FL. Process capability analysis of non-normal process data using the Burr XII distribution. Int J Adv Manuf Technol 2006; 27: 975-984.

[8] Wu HH, Swain JJ, Farrington PA, Messimer SH. A weighted variance capability index for general non-normal processes. Qual Reliab Engng Int 1999; 15: 397-402.

[9] Choobineh F, Branting D. A simple approximation for semivariance. Eur J Oper Res 1986; 27: 364 370.

[10] Chang YS, Choi IS, Bai DS. Process capability indices for skewed populations. Qual Reliab Engng Int 2002; 18: 383-393.

[11] Tang LC, Than SE. Computing process capability indices for non-normal data: a review and comparative study. Qual Reliab Engng Int 1999; 15: 339-353.

[12] Kovarik M, Sarga L. Process capability indices for non-normal data. Wseas Transactions on Business and Economics 2014; 11: 419-429.

[13] Sennaroglu B, Senvar O. Performance comparison of Box-Cox transformation and weighted variance methods with Weibull distribution. Journal of Aeronautics and Space Technologies 2015; 8: 49-55.

[14] Hosseinifard SZ, Abbasi B, Ahmad S, Abdollahian M. A transformation technique to estimate the process capability index for non-normal processes. Int J Adv Manuf Technol 2009; 40: 512-517.

[15] Kotz S, Johnson NL. Process capability indices-a review, 1992-2000 (with discussions). J Qual Technol 2002; 34: 2-53. 
[16] Anis MZ. Basic process capability indices: an expository review. Int Stat Rev 2008; 76:3, 347-367.

[17] Wu CW, Pearn WL, Kotz S. An overview of theory and practice on process capability indices for quality assurance. Int J Prod Econ 2009; 117: 338-359.

[18] Crow EL, M.M. Siddiqui MM. Robust estimation of location. J Am Stat Assoc 1967, 62: 353-389.

[19] Kim T, White H. On more robust estimation of skewness and kurtosis. Financ Res Lett 2004; 1: 65-70.

[20] Bowley AL. Elements of Statistics. Scribner's, New York, 1920.

[21] Hinkley DV. On power transformations to symmetry. Biometrika 1975; 62: 101-111.

[22] Groeneveld RA, Meeden G. Measuring skewness and kurtosis. The Statistician 1984; 33: 391-399.

[23] Kendall MG, Stuart A. The Advanced Theory of Statistics. Griffin, London, 1977.

[24] Moors JJA. A quantile alternative for kurtosis. The Statistician 1988; 37: 25-32.

[25] Hogg RV. More light on the kurtosis and related statistics. J Am Stat Assoc 1972, 67: 422-424.

[26] Hogg RV. Adaptive robust procedures: A partial review and some suggestions for future applications and theory. J Am Stat Assoc 1974, 69: 909-923.

[27] Rivera LAR, Hubele NF, Lawrence FP. $\mathrm{C}_{\mathrm{pk}}$ index estimation using data transformation. Comput Ind Eng 1995, 29: 55:58. 